\title{
Effect of integrative nursing practices on cognitive recovery among severe traumatic brain injury patients
}

\author{
Sahar Younes Othman ${ }^{1}$, Alaa Mostafa Mohamed ${ }^{1}$, Azza Hamdi El-Soussi ${ }^{2}$, Sherif Ahmed Abd El-Monaem ${ }^{3}$, Fatma \\ Refaat Ahmed*2 \\ ${ }^{1}$ Faculty of Nursing, Damanhour University, Egypt \\ ${ }^{2}$ Faculty of Nursing, Alexandria University, Egypt \\ ${ }^{3}$ Faculty of Medicine, Alexandria University, Egypt
}

Received: May 15, 2020

Accepted: June 27, 2020

Online Published: July 13, 2020

DOI: $10.5430 /$ jnep.v10n10p75

URL: https://doi.org/10.5430/jnep.v10n10p75

\begin{abstract}
Background and objective: Cognitive recovery after severe traumatic brain injury (TBI) is the biggest challenge facing the critical care nurses (CCNs). Several studies have been reported that the majority of CCNs are concerned with providing conventional nursing practices to severe TBI patients. These practices are directed to stabilize patients' status rather than to enhance cognitive recovery after TBI. Application of integrative nursing practices for TBI patients can help in enhancing cognitive recovery. Aim: This study aimed to determine the effect of integrative nursing practices on cognitive recovery among severe traumatic brain injury patients.

Methods: A quasi experimental research design was utilized. A convenience sample was conducted on 60 adult patients suffering from severe TBI who admitted to the intensive care units of Alexandria Main University Hospital. Patients were assigned into study group (30 patients) and control group (30 patients). One tool was used for data collection namely "Cognitive Recovery Assessment Tool". This tool was used to assess recovery of cognitive function domains. It consists of level of consciousness using Full Outline of Un-Responsiveness (FOUR) score, level of cognitive functioning using Rancho Los Amigos (RLA) scale and basic cognitive sensory recovery assessment using Western Neuro Sensory Stimulation Profile (WNSSP) scale.

Results: The implementation of integrative nursing practices for the study group was associated with statistically significant positive effects. Patients in the study group showed higher means of consciousness revealed by FOUR score and cognitive function revealed by RLA scale. The basic cognitive sensory recovery revealed by WNSSP scale in the study group was also significantly improved.

Conclusions: Implementation of integrative nursing practices has been shown to enhance cognitive recovery among severe TBI patients. Thus, it is recommended for use in a nurse's daily routine of care for severe TBI patients.
\end{abstract}

Key Words: Cognitive recovery, Integrative nursing practices, Traumatic brain injury

\section{INTRODUCTION}

The brain is an amazing organ that is largely composed of maps and arrays of neurons communicating with each other in trillions of connections called synapses. These neurons represent entire aspects of cognition, or at least entire sensory or cognitive qualities of those aspects. Cognition is the human ability to think, feel, and act. It is the process starting from sensory stimulation transmitted to neurons in the

\footnotetext{
*Correspondence: Fatma Refaat Ahmed; Email: fatmarefaat1312@gmail.com; Address: Faculty of Nursing, Alexandria University, Edmon Fremon St. Smouha, Alexandria, Egypt.
} 
cerebral cortex to a message in the form of nerve impulses from the brain to motor neurons. The complexity of the connectivity between neural networks is mind-boggling which makes any injury to the brain can result in a wide range of cognitive functions impairment. ${ }^{[1,2]}$

Traumatic brain injury (TBI) is the most common devastating traumatic event involving the central nervous system. It is defined as an acute and sudden insult to the brain resulting in alteration and disruption in neural networks and accordingly in normal cognitive functions. TBI is a result of falls, motor vehicle accidents (MVA), being struck by an object, and assaults. ${ }^{[3,4]}$

According to the World Health Organization (WHO), TBI will exceed many diseases as the major cause of death and disability in young adults by the year 2020 . Acute and sudden onset of TBI makes it serious and challengeable health problem in intensive care unit (ICU). It is reported that over 2.5 million new cases of TBI in the United States (U.S.) annually, with 52,000 deaths, and 235,000 of whom are serious enough to require ICU care. Although, the burden of TBI is manifest throughout the world, it is more prominent in developing countries which have inadequately prepared health systems to address the associated health outcomes. In Egypt, according to global burden of diseases (GBD) study 2016, there were 262,264 new cases of TBI and the number of prevalent cases who are lived with TBI related disabilities were 484,935. Additionally, it was found that from 1990 to 2016 , the incidence rates of TBI increased by $24.6 \%$, while prevalence of TBI related disabilities increased by $23.2 \% .^{[5,6]}$

Patients with TBI may present a wide range of cognitive impairment as most of cognitive functions encompass immediate interaction of neural maps and arrays from many different parts of the brain. Severe TBI patients with cognitive impairment are more vulnerable to a wide variety of complications. These complications usually increase ICU length of stay, duration of mechanical ventilation, and morbidity and mortality rates among TBI patients in ICU. Moreover, it creates a major obstacle for re-entry of TBI patients into the community. ${ }^{[7]}$ The cognitive squeals of TBI are determined mainly by TBI severity. Rabinowitz and Levin (2014) ${ }^{[8]}$ found that $65 \%$ of moderate to severe TBI patients report problems with cognitive functioning and may have persisting impairment that limits their ability to return to previous levels of functioning.

Cognitive impairment after severe TBI involves various cognitive domains. These domains include arousal, perception, attention and speed of information processing, verbal and visuospatial skills, psychomotor skills, learning and memory. In addition, it includes executive functions which are the abil- ity to plan, organize, strategize, and focus attention. Deficit in executive functions following TBI can be profound and debilitating, and also have the strongest effect on functional outcomes. ${ }^{[9,10]}$

Cognitive recovery is the biggest challenges facing clinicians following TBI, as TBI patients are often sensitive to the side effects of pharmacological agents. A new paradigm of medical care, known as integrative therapies, is increasing in popularity in managing cognitive impairment after TBI. The approach of this new paradigm is based on the idea that an enriched environment enhances brain plasticity. Brain plasticity is the extraordinary brain's ability to form new neural connections and to modify its own functions following changes in the external environment. So, brain plasticity accelerates cognitive recovery by allowing the brain neurons to compensate for injury. ${ }^{[11-13]}$

Many of integrative therapies fall within the scope of nursing practice, as from its earliest beginnings, nursing has been a holistic discipline focused on the whole person. Integrative nursing is defined as healing-oriented discipline that takes account of the whole person as well as all aspects of lifestyle. Integrative therapies such as music therapy, massage therapy, and aromatherapy to alleviate cognitive impairment have been investigated recently. ${ }^{[14-16]}$

Several studies from the fields of neuromusicology and music cognition have investigated the role of music therapy in cognitive recovery among TBI patients. They reported that music is one of the best cognitive exercises. Moreover, they recommended neurologic music therapy (NMT) as a systematic treatment method to improve cognitive domains of functioning following TBI. It stimulates the affected neural networks, accelerates brain plasticity, and prevents sensory deprivation and accordingly improve TBI patient's cognitive recovery. ${ }^{[17-19]}$

Massage therapy is an ancient therapeutic technique encompasses a variety of hands on techniques all of which are designed to promote self-healing. Massage therapy improves blood flow and the movement of lymph fluid, speeding the removal of metabolic waste products resulting from inactivity, and allowing more oxygen and nutrients to reach the cells and tissues. Which in turn act on tactile sensory receptors within the skin so that the muscles can begin to wake up, bringing to the patient a new awareness to parts of the body that have been cut off as a result of severe TBI . Critical care nurses (CCNs) have to choose the correct type and technique of massage to be used. CCNs play an integral role in choosing the most appropriate aromatic essential oil that serves to achieve the desired effects. With regular usage, effective 
technique, and when provided by a skilled licensed professional CCNs, severe TBI patients could realize cognitive benefits from massage. ${ }^{[20-22]}$

Kohanpour, Peeri and Azarbayjani (2017) $)^{[23]}$ and Wu et al. $(2014)^{[24]}$ found that aromatherapy using lavender oil could lead to improvement in neural plasticity and cognitive functions with significant increase in brain-derived neurotrophic factor (BDNF) levels. These findings may be linked to the small size and fat solubility of lavender oil's molecules which make it able to pass across the blood brain barrier (BBB) near the olfactory nerve. In the body, the only place where the brain is directly exposed to the environment is the olfactory membrane. This deeper penetration of lavender oil in the brain can help in cognitive recovery following TBI. ${ }^{[25,26]}$

Critical care nurses have a unique and trusted role to embrace this holistic focus and to promote safe incorporation of such integrative nursing practices with the conventional nursing practices into the TBI patients' plan of care in a manner like no other health care provider can do. CCNs must ensure the physiological stability of TBI patients before the implementation of such integrative nursing practices. Additionally, CCNs should be able to monitor the patients' autonomic responses while implementing the integrative nursing practices for early detection of any signs of deterioration or to ensure stability or improvement of the patients' status. ${ }^{\text {[27-29] }}$

Furthermore, CCNs must be able to use standardized system of assessing the recovery of TBI patients' cognitive function by using accurate scales. The most common developed and validated scales used in the critical and rehabilitation settings are the Western Neuro Sensory Stimulation Profile (WNSSP), the Sensory Modality Assessment and Rehabilitation Technique (SMART), the Glasgow Coma Scale (GCS), the Full Outline of UnResponsiveness (FOUR) score, and the Rancho Los Amigos (RLA) scale. ${ }^{[30,31]}$

\subsection{Significance of the study}

Despite several studies ${ }^{[32-34]}$ have been conducted to study the effect of integrative nursing practices on improving sleep quality and alleviating pain, stress, and anxiety among ICU patients, the effect of these practices have not been extensively studied on cognitive recovery. Moreover, the national and international studies that examined the effect of such integrative practices did not focus on the TBI patients rather than focusing on ICU patients with cognitive impairment whatever the cause. ${ }^{[35,36]}$ Thus, further studies are needed to be conducted in this area to determine the effect of implementing integrative nursing practices on cognitive recovery among severe TBI Patients.

Published by Sciedu Press

\subsection{Aim of the study}

This study aims to determine the effect of integrative nursing practices on cognitive recovery among severe traumatic brain injury patients.

\subsection{Research hypotheses}

H1. Severe TBI patients who are subjected to integrative nursing practices exhibit higher level of consciousness than those who are not subjected.

H2. Severe TBI patients who are subjected to integrative nursing practices exhibit higher level of cognitive function than those who are not subjected.

H3. Severe TBI patients who are subjected to integrative nursing practices exhibit higher basic cognitive sensory recovery than those who are not subjected.

\section{MATERIALS AND METHOD}

\subsection{Research design}

A quasi experimental research design was utilized to accomplish this study.

\subsection{Setting}

This study was conducted at Alexandria Main University Hospital (AMUH) ICUs namely; casualty care unit (unit I), general ICUs (unit II, unit III, unit IV). These units receive patients with different disorders in acute stage of illness or severe injuries. They are prepared to receive patients directly from the emergency department or transferred from any other hospitals.

\subsection{Subjects}

The study sample size was calculated by power analysis (Epiinfo program); expected frequency $=50 \%$, acceptable error $=5 \%$, confidence coefficient $=95 \%$, minimum sample size $=56$ in which power analysis data $=0.8$. Accordingly, $\mathrm{a}$ convenience sample of $60 \mathrm{TBI}$ patients who were admitted to the previously mentioned setting was included in this study. The subjects were recruited according to the following inclusion and exclusion criteria. Inclusion Criteria: Patients were eligible to the study if they were unconscious $>72$ hours post injury with a GCS score of 3-8, hemodynamically stable, and aged from 21 to 60 years of both sexes. Patients were excluded from the study based on the following exclusion criteria: receiving sensory suppression medications (sedatives, muscle relaxant), suffering from blindness, deafness, and seizures or patients who had brain stem death. Random assignment of the studied patients into two groups; control and study group (30 patients in each). The control group received the conventional nursing care for severe TBI provided by the $\mathrm{CCNs}$, while the study group received the integrative nursing practices provided by the researcher. 


\subsection{Tool}

One tool was used to collect the data of this study namely "Cognitive Recovery Assessment Tool". This tool was used to assess recovery of cognitive function domains. It consists of four parts:

Part I: Patient's demographic and baseline characteristics.

This part was developed by the researchers to collect demographic data such as sex, age and clinical data such as GCS score, date of admission, mechanism of injury, accompanying injuries, past medical history for sensory motor deficits.

Part II: Level of consciousness assessment using FOUR score.

The Full Outline of UnResponsiveness score (FOUR score) was adopted from Wijdicks et al. (2005) ${ }^{[37]}$ to assess level of consciousness and arousal domains of function in the studied patients. FOUR score is a 17-point scale range from 0 to 16. The FOUR score assesses four domains of neurological function: eye responses, motor responses, brainstem reflexes, and respiration pattern. The maximum score for the four items is 4 . In which 0 assumes the absence of breathing and brainstem reflexes, while 16 indicates full consciousness.

Part III: Level of cognitive function assessment using Rancho Los Amigos scale.

The Rancho Los Amigos (RLA) scale was adopted from Hagen et al. (1972) ${ }^{[38]}$ to evaluate deficits in the studied patient's cognitive function especially in executive functions domain. This scale consists of eight levels. Level I (no response, needs total assistance), level II (generalized response, needs total assistance), level III (localized response, needs total assistance), level IV (confused/agitated, needs maximal assistance), level $\mathrm{V}$ (confused, inappropriate, non-agitated, needs maximal assistance), level VI (confused-appropriate, needs moderate assistance), level VII (automatic-appropriate, needs minimal assistance), level VIII (purposeful-appropriate, needs stand by assistance).

Part IV: Basic cognitive sensory recovery assessment using WNSSP scale.

The Western Neuro Sensory Stimulation Profile scale (WNSSP scale) was adopted from Ansell and Keenan (1989), ${ }^{[39]}$ to assess the studied patient's recovery of basic cognitive sensory function domains in response to sensory stimulation. This scale had 32 items that assess patients' arousal/attention, expressive communication, and response to auditory, visual, tactile, and olfactory stimulation. All items were scored using multipoint systems that ranged from 0-1 to $0-5$ and varied from item to item. The minimum score is 0 and the maximum score was 110. Low scores of WNSSP indicate poorer cognitive functions.

\subsection{Validity and reliability of tool}

The study tool was reviewed by 7 experts in the field of critical care nursing to test its content validity and ascertain that the study tool was relevant, and applicable. The reliability and validity of FOUR score were approved by Iyer et al. (2009) ${ }^{[40]}$ (Kappa coefficient of 0.99). The reliability of the RLA scale was assessed by McCauley et al. (2010) ${ }^{[41]}$ and a Kappa coefficient of 0.92 was reported. Concerning the reliability of WNSSP scale, it was previously tested by Cusick et al. (2014) ${ }^{[30]}$ and indicated significant reliability of (0.93).

\subsection{Study procedure}

\subsubsection{Administrative and ethical considerations}

The Ethics Committee of the Faculty of Nursing at the Alexandria University was approved the current study. Further, a permission to conduct the current study was taken from the hospital authorities after explanation of the aim of the study. Before data collection the researcher (the second author) attended an extensive therapeutic massage training program. This program was certified from Health Sciences Department, Faculty of Physical Education, Alexandria University. There were no threats that can affect the patients during the study implementation. The safe use of cold pressed lavender oil either by inhalation or skin massage was ensured from Botany and Microbiology Department, Faculty of Science, Alexandria University before the study implementation. A code number was assigned to each patient to ensure confidentiality. The researcher asked the patient's relative about his/her acceptance of patient's participation in the study. Each relative signed a written consent. Patients' relatives were also assured that the quality of care provided would not be affected if they did not wish to participate in the study. The ethical demand of doing well, being fair, and not harming, thereby appeared to have been fulfilled for both control and study groups.

\subsubsection{A pilot study}

The pilot study was performed on 6 severe TBI patients (10\%) before starting data collection. After that, those patients were excluded from the total sample. The aim of the pilot study was to determine the clarity, applicability, feasibility and the required time to fulfill the designed tool. Then accordingly necessary modifications were done. Pilot study revealed that further modifications were not needed.

\subsection{Data collection}

Data were collected over a period of nine consecutive months (from July 2018 to March 2019). All the eligible patients were assigned to two studied groups. Those assigned to the control group received the conventional nursing care for severe TBI provided by the CCNs $(n=30)$, while those in the 
study group received the integrative nursing practices $(\mathrm{n}=$ 30). Data were collected from the control group first and after its completion, it was collected from the study group.

For both groups:

Eligible patients were approached in the fourth day post injury to ensure hemodynamic stability. The researcher selected 2 hours between 3 pm and 9 pm to avoid any conflict with medical rounds or nursing care provided to the patients. ${ }^{[42]}$ The demographic and clinical data of the studied patients were collected from the patient's chart or from the patient's family and recorded. The baseline data of level of consciousness (LOC), level of cognitive functions (LCF), and basic cognitive sensory recovery were assessed at the 1 st day of the study using the study tool.

For the control group:

Patients were subjected to the conventional nursing care provided by CCNs in the study setting without the researcher's interference. The researcher observed the conventional nursing stimulation practices that may affect the patients' cognitive recovery for 90 minutes per day for four consecutive days, from the fourth day to the seventh day post injury. These practices include: tactile stimulation such as changing position, auditory stimulation such as play Qur'an beside the patient, olfactory stimulation such as performing oral care, gustatory stimulation such as performing gum message, and visual stimulation such as performing eye care.

For the study group:

The integrative nursing practices; massage therapy, aromatherapy, and neurologic music therapy were carried out for 90 minutes per day for four consecutive days, from the fourth day to the seventh day post injury. The integrative nursing practices were performed in the following steps:

1) Awakening the TBI patient; integrative nursing practices were preceded by 10 minutes awakening period to stimulate patient's five senses, during this awakening period the researcher introduced herself to the patient and spoke to him explaining what will be done. Then, the researcher opened the patient's eyes and kept them open with one hand. While, at the same time, moistened the patient's face with a wet gauze, then put a mirror in front of the patient's face to see himself. Further, the researcher put a small amount of coffee near the patient's nose. Finally, the researcher put $2 \mathrm{ml}$ lemon juice (acidic solution) in the patient's mouth by a syringe. ${ }^{[42]}$ 2) Massage therapy; each patient in the study group was massaged by the researcher during a 40 minutes session. Lavender oil was used for effleurage and massage facilitation and as a part of aromatherapy. Five drops of lavender oil was poured into researcher's hands and was warmed by holding it for a few seconds before beginning the rub.
Massage techniques included static massage, squeezing massage, stretching massage, vibratory massage, and transverse friction massage. Areas with inflammation, petechiae, ecchymosis, subcutaneous hemorrhage, or wounds were not be massaged. ${ }^{[35,36,43,44]}$ Each massage session was carried out as the following:

- Back massage technique (Approximately 10 Minutes): The researcher massaged the middle of the patient's lower back in circular motions. The areas over right and left shoulder blades were stroked upward and massaged again using circular motion. The same areas were stroked downward and ended by massaging the iliac crests. These processes were repeated for three times.

- Feet and legs massage technique (Approximately 10 Minutes): The researcher used both hands to effleurage up lower leg and petrissage medial aspect of lower leg. The researcher also used her thumb to glide up lateral aspect of lower leg, then performed figure of eight around knee using both hands and loose fist on quadriceps. The researcher repeated this massage technique three times.

- Abdominals massage technique (Approximately 4 Minutes): The researcher massaged the abdomen in the direction of peristalsis or in a clockwise direction. The researcher then slid her hands with gentle; even pressure up the middle of patient's abdomen until felt the patient's ribs. The researcher put both hands on the lower right side of the abdomen. Then moved her palms up to the top of the navel until reached the patient's ribs. The researcher swept hands across the abdomen just below the ribs and down to the lower left side of patient's abdomen. The navel circling massage was repeated three times.

- Hands and Arms massage technique (Approximately 10 Minutes): The researcher gently rubbed over the metacarpals in circular motion. Then gentle stretches were performed to open the patient's hand by the researcher's fingers. The researcher stretched out the patient's fingers and performed circular rubbing and then stroked along the bones of the patient's palm. Both sides of the forearm gently were stroked back towards the heart using thumb glide. Then, shoulder stretch and traction were performed. Both hands and arms massage were done three times each session.

- Chest massage technique (Approximately 5 Minutes): The researcher slid flat hands firmly but gently up to the middle of patient's chest. Before researcher's hands reached the collarbones, fanned hands out toward the patient's shoulders, applied gentle, but firm 
pressure to the muscles in the upper chest. Once researcher's hands reached the patient's shoulder, pushed them over under the shoulder area, then back up over the upper arm. From the upper arm, researcher's hands moved into the ribcage area, started at the armpits. Researcher's hands were slid down the length of the rib cage. After that researcher's hands pulled back up into the chest area from where researcher began. Massage was repeated three times.

- Gum massage technique (Approximately 1 Minutes): The researcher placed thumb on the outer gum line and the index finger on the inner gum line, directly behind the thumb. Then patient's gum was gently be massaged in a circular motion.

3) Aromatherapy: In the current study, lavender oil was used for aromatherapy. Lavender oil was prepared as a cold pressed oil under the supervision of professor in Faculty of Science; Alexandria University specialized in Botany and Microbiology. In aromatherapy procedure a cotton gauze $(2 \mathrm{~cm} \times 2 \mathrm{~cm})$ with 5 drops of lavender oil was introduced to the patients in the study group through attaching it to the front of the patient's gown, approximately 12 inches below their nose for 10 minutes.

4) Neurologic music therapy; patients in the study group listened to 30 minutes of brain stimulating music symphonies after implementation of massage and aromatherapy (Hegde, 2014). ${ }^{[19]}$ The musical symphonies were presented through Sony MDR-XB 450 headphone (amplitude set to approximately $60-80 \mathrm{~dB}$ ). This headphone has the property of noise canceling to prevent external noise interference.

For both groups (after integrative nursing practices/conventional nursing care):

The LOC, LCF, and basic cognitive sensory recovery were assessed using the study tool after each implementation of the integrative nursing practices for the study group and after 90 minutes of observation of conventional nursing stimulation practices for the control group throughout the four days of the study period to be compared with the baseline data.

\subsection{Statistical analysis}

1) The collected data were coded and entered in special format to be suitable for computer feeding.

2) Following data entry, checking and verification process were carried out in order to avoid any errors.

3) Data were analyzed using the statistical package for social science SPSS (version 20).

4) The following statistical analysis measures were used:

- Descriptive statistical measures, which included: numbers, percentages, and averages (Minimum, Maximum, Arithmetic mean $(\bar{X})$, Standard deviation (SD).
- Statistical analysis tests, which included: Chi square, Fisher Exact test (when more than $20 \%$ of the cells had expected count less than 5, correction for chi-square test was conducted by fisher's exact test), student $t$ test and paired $t$ test.

- Graphical presentation included: Bar graphs were done for data visualization.

\section{RESULTS}

Table 1 represents the distribution of the studied groups according to demographic and clinical characteristics. Concerning patients' sex, this table shows that $73.3 \%$ of patients in the control group were male compared to $86.6 \%$ of the study group. In relation to the age, it can be noted that half of the patients in the study and control group were between 21-30 years. The mean age was $33.97 \pm 11.54$ and 33.83 \pm 13.88 years for patients in the study and control groups respectively. As regards the mechanism of injury, the high percentages of patients in both groups were injured by motor vehicle accident; representing $73.3 \%$ for patients in the control group and $60 \%$ for the study group. This table also shows that $66.7 \%$ and $80 \%$ of patients in the control and study groups respectively had accompanying injuries along with TBI. Concerning the GCS, it was found that the mean GCS was $5.73 \pm 1.26$ for patients in the control group and $6.0 \pm 1.29$ for patients in the study group. In relation to the CT scan findings, it can be noted that the highest percent was for subdural hematoma in both groups, it accounts for $46.7 \%$ of the control group and $56.7 \%$ of the study group. This table also presents that half of patients in the control group and $70 \%$ of patients in the study group were managed medically only. It can be noted from the same table that both groups of patients (control and study), related to demographics and clinical characteristics, were comparable with no significant difference were noted; in other words, both groups would be considered homogeneous.

Table 2 reflects comparison between the control and study groups according to mean differences of the LOC scores at the start and end of the study (using FOUR score). It can be noted that there were no significant differences in the mean scores of all LOC domains between the control and study groups at the start of the study. At the end of the study, it can be observed that there was a highly significant differences between the two groups in relation the eye and motor response $p=.000$. Also, in relation to brain reflexes and respiration domains, it was found that there was a significant difference between the two studied groups $p=.046$ and $p=.024$ respectively. Additionally, it can be detected from the same table that the mean LOC score for the control group was $6.67 \pm$ 2.28 compared to $7.20 \pm 2.34$ for the study group with no 
significant difference between the two groups at the start of the study $(p=.378)$. At the end of the study, these means increased to $7.40 \pm 1.85$ for patients in the control group and to $9.67 \pm 2.41$ for the study group with a high significant difference between the two groups $(p=.000)$. As shown in
Figure 1, patients in the study group exhibited higher mean scores of LOC all over the study period (along four days) with accomplishment of integrative nursing practices compared to patients in the control group after application of conventional nursing care.

Table 1. Socio-demographic data and clinical characteristics of the studied groups

\begin{tabular}{|c|c|c|c|c|c|c|}
\hline \multirow{3}{*}{$\begin{array}{l}\text { Demographic and clinical } \\
\text { characteristics }\end{array}$} & \multicolumn{4}{|c|}{ Studied groups } & \multirow{3}{*}{ Test of Sig. } & \multirow{3}{*}{$p$} \\
\hline & \multicolumn{2}{|c|}{$\begin{array}{l}\text { Control } \\
(n=30)\end{array}$} & \multicolumn{2}{|c|}{$\begin{array}{l}\text { Study } \\
(\mathrm{n}=30)\end{array}$} & & \\
\hline & No. & $\%$ & No. & $\%$ & & \\
\hline \multicolumn{7}{|l|}{ Sex } \\
\hline Male & 22 & 73.3 & 24 & 80.0 & \multirow[t]{2}{*}{$\chi^{2}=0.373$} & \multirow[t]{2}{*}{.542} \\
\hline Female & 8 & 26.7 & 6 & 20.0 & & \\
\hline \multicolumn{7}{|l|}{ Age (years) } \\
\hline $21-30$ & 15 & 50.0 & 15 & 50.0 & \multirow{4}{*}{$\chi^{2}=1.887$} & \multirow{4}{*}{.634} \\
\hline $31-40$ & 6 & 20.0 & 9 & 30.0 & & \\
\hline $41-50$ & 5 & 16.7 & 2 & 6.7 & & \\
\hline $51-60$ & 4 & 13.3 & 4 & 13.3 & & \\
\hline Mean \pm SD & \multicolumn{2}{|c|}{$33.97 \pm 11.54$} & \multicolumn{2}{|c|}{$33.83 \pm 13.88$} & $t=0.040$ & .968 \\
\hline \multicolumn{7}{|l|}{ Mechanism of injury } \\
\hline Motor vehicle accident & 22 & 73.3 & 18 & 60.0 & $\chi^{2}=1.200$ & .273 \\
\hline Fall from height & 8 & 26.6 & 9 & 30.0 & $\chi^{2}=0.800$ & .371 \\
\hline Assault & 2 & 6.7 & 3 & 10.0 & $\chi^{2}=0.218$ & ${ }^{\mathrm{FE}} p=1.000$ \\
\hline Hard object hit the head & 1 & 3.4 & 0 & 0.0 & $\chi^{2}=3.158$ & ${ }^{\mathrm{FE}} p=0.237$ \\
\hline \multicolumn{7}{|l|}{ Accompanying injuries } \\
\hline Yes & 20 & 66.7 & 24 & 80.0 & \multirow{2}{*}{$\chi^{2}=1.364$} & \multirow{2}{*}{.243} \\
\hline No & 10 & 33.3 & 6 & 20.0 & & \\
\hline GCS score Mean \pm SD. & \multicolumn{2}{|c|}{$5.73 \pm 1.26$} & \multicolumn{2}{|c|}{$6.0 \pm 1.29$} & $t=0.812$ & .420 \\
\hline \multicolumn{7}{|l|}{ CT scan findings } \\
\hline Subdural Hematoma (SDH) & 14 & 46.7 & 17 & 56.7 & $\chi^{2}=0.601$ & .438 \\
\hline Epidural Hematoma (EDH) & 2 & 6.7 & 4 & 13.3 & $\chi^{2}=0.000$ & ${ }^{\mathrm{FE}} p=1.000$ \\
\hline $\mathrm{ICH}$ and brain contusion & 7 & 23.3 & 6 & 20.0 & $\chi^{2}=1.002$ & .317 \\
\hline Simultaneous SDH and EDH & 7 & 23.3 & 3 & 10.0 & $\chi^{2}=3.268$ & .145 \\
\hline Other & 24 & 80.0 & 22 & 73.3 & $\chi^{2}=0.373$ & .542 \\
\hline \multicolumn{7}{|l|}{ Types of management } \\
\hline Medical & 15 & 50.0 & 21 & 70.0 & $\chi^{2}=2.500$ & .114 \\
\hline Both medical and surgical & 15 & 50.0 & 9 & 30.0 & & \\
\hline
\end{tabular}

Table 3 represents comparison between the control and study groups according to mean differences of the level of cognitive function scores (LCF) at the start and end of the study (using RLA scale). For patients in the study group, the mean score of LCF was increased from $2.80 \pm 1.24$ at the start of the study (pre-application of integrative nursing practices) to $5.13 \pm 1.53$ at the end of the study (after application of integrative nursing practices). While patients in the control group, the mean score was $2.77 \pm 1.19$ at the start of the Published by Sciedu Press study and $2.80 \pm 1.24$ at the end of the study. Also, it can be noted that there was no significant difference between the two groups in relation to the mean scores of LCF at the start of the study ( $p=.925)$, whereas there was a highly significant difference between them at the end of the study $(p=.000)$. As displayed in Figure 2, despite that both groups were similar at the start of the study, patients in the study group had a higher LCF scores all over the study period (along four days) than patients in the control group. 
Table 2. Comparison between the control and study groups according to mean differences of the level of consciousness scores at the start and end of the study

\begin{tabular}{|c|c|c|c|c|}
\hline \multirow{2}{*}{$\begin{array}{l}\text { Level of Consciousness Domains } \\
\text { Using FOUR Score }\end{array}$} & \multirow{2}{*}{ Time } & \multicolumn{2}{|l|}{ Studied groups } & \multirow{2}{*}{$t\left(p^{2}\right)$} \\
\hline & & Control $(n=30)$ & Study $(\mathrm{n}=\mathbf{3 0})$ & \\
\hline \multirow{2}{*}{ Eye response } & Start & $0.93 \pm 0.78$ & $1.07 \pm 0.87$ & $0.656(.514)$ \\
\hline & End & $0.93 \pm 0.83$ & $2.67 \pm 1.18$ & $6.606\left(.000^{*}\right)$ \\
\hline$t\left(p^{1}\right)$ & & --- & $5.978\left(.000^{*}\right)$ & \\
\hline \multirow{2}{*}{ Motor response } & Start & $2.27 \pm 0.98$ & $2.53 \pm 1.04$ & 0.997 (.323) \\
\hline & End & $2.20 \pm 1.06$ & $3.13 \pm 0.86$ & $3.732\left(.000^{*}\right)$ \\
\hline$t\left(p^{1}\right)$ & & $0.266(.792)$ & $2.435\left(.018^{*}\right)$ & \\
\hline \multirow{2}{*}{ Brainstem reflexes } & Start & $3.47 \pm 1.07$ & $3.57 \pm 1.19$ & $0.342(.733)$ \\
\hline & End & $3.37 \pm 1.13$ & $3.87 \pm 0.73$ & $2.036\left(.046^{*}\right)$ \\
\hline$t\left(p^{1}\right)$ & & $0.352(.726)$ & $1.177(.244)$ & \\
\hline \multirow{2}{*}{ Respiration } & Start & $0.93 \pm 0.25$ & $0.90 \pm 0.31$ & $0.413(.681)$ \\
\hline & End & $0.97 \pm 0.32$ & $1.43 \pm 1.04$ & $2.316(.024 *)$ \\
\hline$t\left(p^{1}\right)$ & & $0.539(.592)$ & $2.675(.009 *)$ & \\
\hline Total FOUR Score & Start & $6.67 \pm 2.28$ & $7.20 \pm 2.34$ & 0.889 (.378) \\
\hline (Mean \pm SD) & End & $7.40 \pm 1.85$ & $9.67 \pm 2.41$ & $4.092\left(.000^{*}\right)$ \\
\hline$t\left(p^{1}\right)$ & & $1.362(.179)$ & $4.028(.000 *)$ & \\
\hline
\end{tabular}

Note. FOUR Scores: Full Outline of Un-Responsiveness Scores; Start: pre intervention (integrative nursing practices)/conventional nursing care $1^{\text {st }}$ day; End: post intervention (integrative nursing practices)/conventional nursing care 4th day; $p^{1}: p$ value for comparing mean FOUR scores between start and end of integrative nursing practices/conventional; $p^{2}: p$ value for comparing total mean differences between control and study groups.

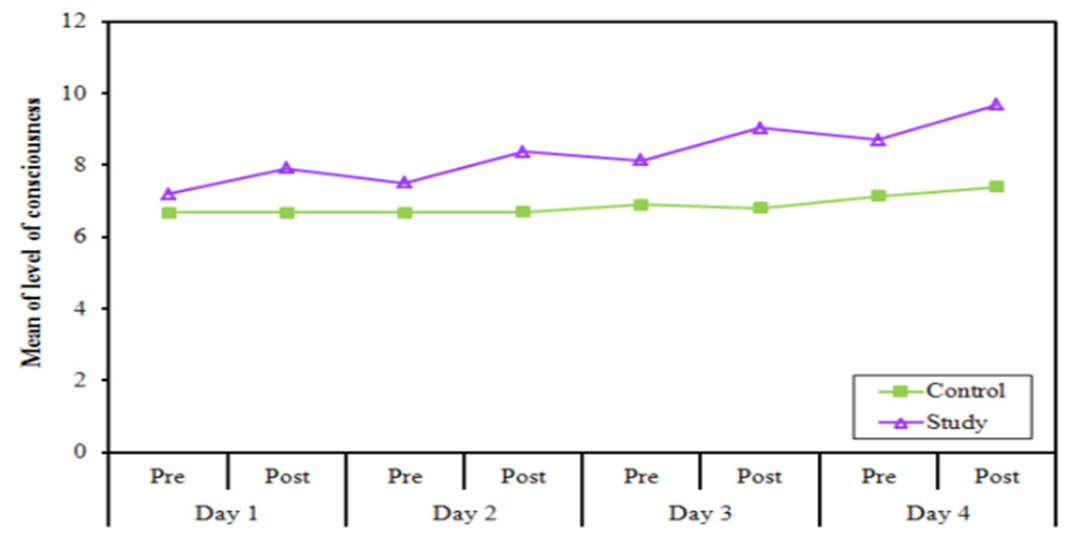

Figure 1. The change of the level of consciousness mean scores all over the study period (four days) in the control and study groups

Table 3. Comparison between the control and study groups according to mean differences of the level of cognitive function scores at the start and end of the study

\begin{tabular}{lllll}
\hline \multirow{2}{*}{ Variable } & \multirow{2}{*}{ Time } & \multicolumn{2}{c}{ Studied groups } & \multirow{2}{*}{$\left(\boldsymbol{p}^{\mathbf{2}}\right)$} \\
\cline { 3 - 5 } & & Control $(\mathbf{n}=\mathbf{3 0})$ & Study $(\mathbf{n}=\mathbf{3 0})$ & \\
\hline Level of Cognitive Function Scores using & Start & $2.77 \pm 1.19$ & $2.80 \pm 1.27$ & $0.094(.925)$ \\
RLA scale & End & $2.83 \pm 1.37$ & $5.13 \pm 1.53$ & $6.134\left(.000^{*}\right)$ \\
$t\left(p^{1}\right)$ & & $0.181(.857)$ & $6.418\left(.000^{*}\right)$ & \\
\hline
\end{tabular}

Note. RLA scale: Rancho Los Amigos scale; Start: pre intervention (integrative nursing practices)/conventional nursing care $1^{\text {st }}$ day; End: post intervention (integrative nursing practices)/conventional nursing care 4th day; $p^{1}: p$ value for comparing mean pain scores between start and end of integrative nursing practices/conventional nursing care. $p^{2}: p$ value for comparing total mean differences between control and study groups; $t$ : student $t$ test; *: Statistically significant at $p \leq .05$. 


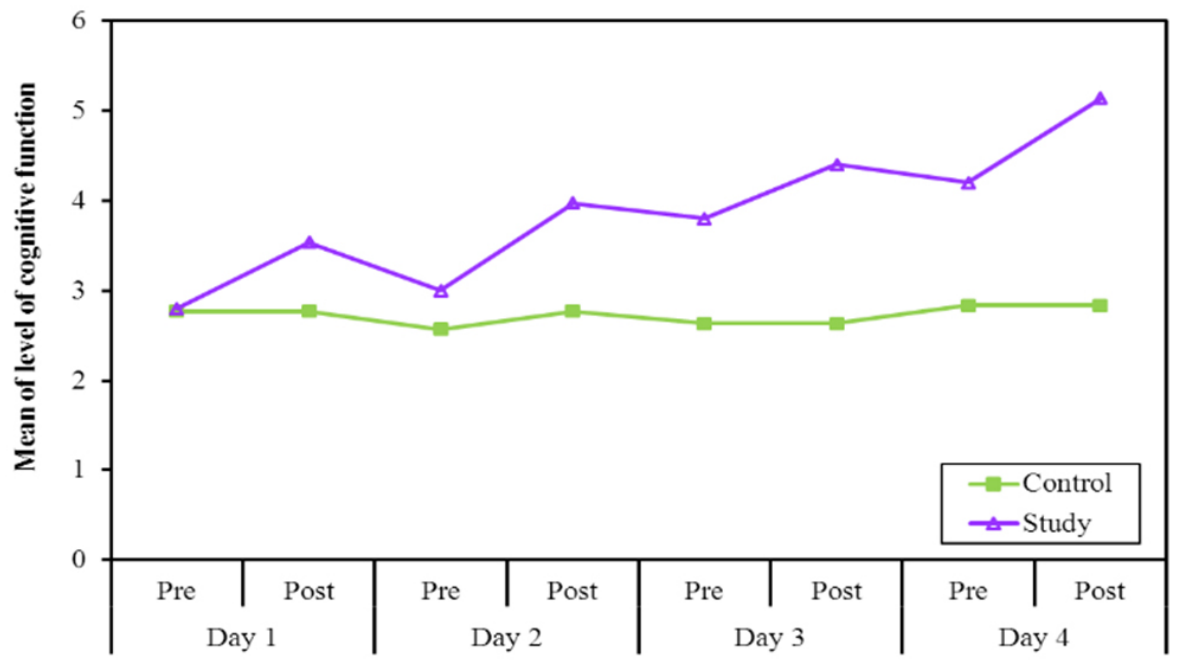

Figure 2. The change of the level of cognitive function mean scores throughout the study period (four days) in the study and control groups

Table 4. Comparison between the control and study groups according to mean differences of the basic cognitive sensory recovery scores at the start and end of the study

\begin{tabular}{lllll}
\hline $\begin{array}{l}\text { Basic Cognitive Sensory Recovery } \\
\text { Domains Using WNSSP Scale }\end{array}$ & \multirow{2}{*}{ Time } & \multicolumn{2}{l}{ Studied groups } & \multirow{2}{*}{$\left(\boldsymbol{p}^{2}\right)$} \\
\cline { 3 - 5 } Arousal/attention & Control $(\mathbf{n}=\mathbf{3 0})$ & Study $(\mathbf{n}=\mathbf{3 0})$ & $2.035\left(.046^{*}\right)$ \\
$t\left(p^{1}\right)$ & Etart & $1.37 \pm 1.22$ & $0.83 \pm 0.79$ & $8.625\left(.000^{*}\right)$ \\
Auditory response & & $1.43 \pm 1.43$ & $5.47 \pm 2.13$ & $1.243(.219)$ \\
$t\left(p^{1}\right)$ & Start & $0.175(.862)$ & $11.187\left(.000^{*}\right)$ & $7.211\left(.000^{*}\right)$ \\
Expressive communication & End & $6.30 \pm 5.95$ & $4.47 \pm 5.44$ & \\
$t\left(p^{1}\right)$ & & $0.146(.885)$ & $8.97 \pm\left(.000^{*}\right)$ & $1.128(.264)$ \\
Visual response & Start & $0.47 \pm 0.73$ & $0.27 \pm 0.64$ & $6.729\left(.000^{*}\right)$ \\
$t\left(p^{1}\right)$ & End & $0.60 \pm 0.93$ & $3.10 \pm 1.81$ & \\
Tactile response & & $0.602(.549)$ & $8.074\left(.000^{*}\right)$ & $1.887(.064)$ \\
$t\left(p^{1}\right)$ & Start & $7.40 \pm 5.61$ & $4.57 \pm 6.0$ & $6.481\left(.000^{*}\right)$ \\
Olfactory response & End & $8.40 \pm 8.06$ & $23.57 \pm 9.97$ & \\
$t\left(p^{1}\right)$ & & $0.558(.579)$ & $8.943\left(.000^{*}\right)$ & $2.212\left(.031^{*}\right)$ \\
Total Score & Start & $4.0 \pm 3.16$ & $2.30 \pm 2.78$ & $6.292\left(.000^{*}\right)$ \\
$($ Mean \pm SD) & End & $3.87 \pm 3.48$ & $10.10 \pm 4.16$ & \\
$t\left(p^{1}\right)$ & & $0.152(.880)$ & $8.539\left(.000^{*}\right)$ & $2.016\left(.048^{*}\right)$ \\
\hline
\end{tabular}

Note. WNSSP Scale: Western Neuro Sensory Stimulation Profile scale; Start: pre intervention (integrative nursing practices)/conventional nursing care $1^{\text {st }}$ day; End: post intervention (integrative nursing practices)/conventional nursing care 4th day; $p^{1}: p$ value for comparing mean WNSSP scores between start and end of integrative nursing practices/conventional nursing care. $p^{2}: p$ value for comparing total mean differences between control and study groups; $t$ : student $t$ test; *: Statistically significant at $p \leq .05$.

Table 4 shows comparison between the control and study groups according to mean difference of basic cognitive sensory recovery scores at the start and end of the study (using
WNSSP scale). At the start of the study, it can be noted that there were no significant differences in the mean scores of auditory expressive communication and visual response 
between patients in the control and study groups ( $p=.219$, .264 , and .064) respectively. While at the end of the study, the mean scores of all cognitive sensory recovery scores (arousal/attention, auditory, expressive communication, visual, tactile, and olfactory response) were increased significantly in the study group compared to the control group ( $p=$ .000). It can be observed from the same table that the total mean score of basic cognitive sensory recovery domains for patients in the control group $(19.90 \pm 15.38)$ was higher than the study group $(12.57 \pm 14.53)$ at the start of the study. While, upon the completion of the study the mean score of basic cognitive sensory recovery domains for patients in the study group $(62.30 \pm 23.96)$ was higher than the control group (20.67 \pm 19.12$)$. Additionally, It was found that there was no significant difference between both groups regarding to the total mean scores of basic cognitive sensory recovery domains at the start of the study $(p=.063)$, whereas there was a high significant difference between them at the end of the study $(p=.000)$. Figure 3 demonstrates the trend of changes in the of basic cognitive sensory recovery mean scores determined by WNSSP scale throughout the study period in the control and study groups. This figure indicates that the mean scores of the WNSSP scale increased in patients of the study group from the first through the fourth day of the study. However, the mean scores of the WNSSP scale in the control group seemed to be steadier than the study group throughout the study period.

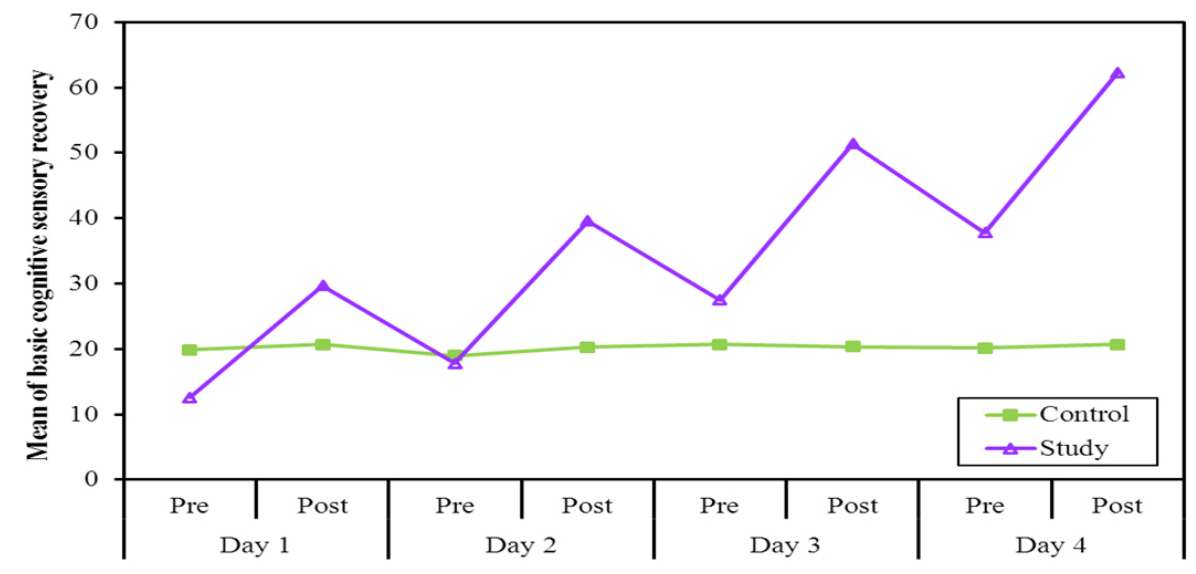

Figure 3. The change of the level of basic cognitive sensory recovery mean scores throughout the study period in the control and study groups

\section{Discussion}

Cognitive recovery after severe TBI is the biggest challenge facing the ICU clinicians particularly the CCNs. Unfortunately, several studies have been reported that the majority of CCNs are concerned with providing conventional nursing care to severe TBI patients such as oral and tracheal tube suction, patient repositioning and maintenance of fluid, electrolytes and nutritional balance. These practices are directed to stabilize patients' status and minimize comorbid complications rather than to enhance cognitive recovery after TBI. ${ }^{[42,45,46]}$

Incorporation of integrative nursing practices in the care provided to TBI patients may potentially stimulate affected neural networks, accelerate brain plasticity, and avoid a sensory deprivation that could slow down the TBI patient's cognitive functions recovery. Environmental enrichment is the main cause behind improvement of cognitive functions associated with application of integrative nursing practices such as NMT, massage, and aromatherapy to severe TBI patients. ${ }^{[15,47]}$ In response to this, the current study was conducted to determine the effect of integrative nursing practices on cognitive recovery among severe traumatic brain injury patients.

The present study shows that most of the studied patients were male; middle aged less than thirty years old. Moreover, the current results reveal that motor vehicle accidents are the main mechanism of injury among the studied patients. Findings of the current study may be attributed to the rapid lifestyle with lack of application of safety precautions among young adult males. These results are supported by Alam et al. (2016) $)^{[48]}$ and El-Fiki and El Falaky (2016) ${ }^{[49]}$ findings.

Cognitive recovery after severe TBI occurs at many cognitive function domains. Improvements in consciousness and arousal level, patients' behaviors that are present in each level of cognitive functions recovery, and basic cognitive sensory recovery can provide a strong reflection of recovery that take place among all cognitive function domains. Often cognitive recovery curve after severe TBI includes many ups, downs 
and plateaus. Implementation of integrative nursing practices after severe TBI has been associated with a steady improvement in LOC, LCF, and basic cognitive sensory recovery throughout the course of cognitive functions recovery. The findings of the present study revealed that the mean values of LOC determined by FOUR score increased significantly in the study group after implementing integrative nursing practices compared to the control group. This improvement in the LOC may be attributed to repetitive auditory, tactile and olfactory stimulation using NMT, massage, and lavender oil. As based on brain plasticity and coma arousal theories, the brain's adaptive capacity to develop new pathways in the injured brain by passing the damaged part of the brain thus reestablishing a pathway to the healthy cells can be enhanced through enriched environment by utilization of multimodal stimulation. Moreover, this improvement can be explained by the music listening effect on activation of a vast bilateral networks of frontothalamic system which are responsible for maintaining arousal and attention. ${ }^{[50,51]}$

These findings are agreed by Alam et al. (2016) ${ }^{[48]}$ and Megha et al. (2013) ${ }^{[52]}$ who investigated the effect of multimodal coma stimulation on the consciousness levels in comatose patients with TBI . They concluded that LOC in the study group were significantly higher than those in the other group. Additionally, Sun and Chen $(2015)^{[53]}$ study to assess the role of music therapy on LOC among severe TBI patients revealed that the LOC were significantly higher in the music group compared to the control group. Similarly, Cevik and Namik (2018) ${ }^{[54]}$ reported significant increase in the mean values of GCS among comatose patients after auditory stimulation application in comparison with GCS of patients in the control group.

Also, in line with the results of the current study, Hatefi et al. (2015) ${ }^{[21]}$ investigated the effect of massage therapy on GCS score in TBI patients in ICU. They found that there were significant increases in the mean values of GCS score in the TBI intervention group who received massage therapy than the control group. Similarly, Jalil et al. (2015) ${ }^{[55]}$ who aimed to determine the effects of aromatic foot massage on the LOC in comatose patients due to TBI. They stated that the mean values of GCS score in the intervention group were improved by the end of the intervention.

Moreover, findings of the current study are in accordance with Wu et al. ( 2014) ${ }^{[24]}$ study to assess the effect of aromatherapy massage on brain electrical activity, their findings revealed that alpha-brain wave activities were significantly enhanced and delta wave activities were markedly reduced following the one-time aromatherapy massage with lavender oil. Similarly, another study conducted by Sayorwan et al. (2012) ${ }^{[25]}$ to assess the effects of lavender oil inhalation on brain electrical activity, they found that lavender oil inhalation increased the power of alpha brain activities.

Contrary to the findings of the current study, Cheng et al. (2018) ${ }^{[56]}$ who conducted a study to investigate the impact of implementing multimodal sensory stimulation on the recovery of consciousness in patients with severe TBI. They concluded that implementing of this stimulation may not be sufficient to restore consciousness in severe TBI patients. Additionally, Moattari et al. (2016) ${ }^{[42]}$ conducted a study to determine the effects of sensory stimulation conducted by nurses and families on the consciousness of head injury comatose patients. They found that there were no significant differences in the mean scores of GCS between the control group and the nurse-based sensory stimulation group.

Concerning the effect of integrative nursing practices on patients' behaviors that are present in each LCF recovery, findings of the current study showed a significant increase in the mean values of RLA scale among patients in the study group compared to the control group. This improvement may be attributed to the neuroplastic effects of multimodal sensory stimulation resulting from application of NMT, massage, and lavender oil which improve reorganization of brain activity, adaptability, and functions. ${ }^{[12]}$ Furthermore, tactile and olfactory stimulation caused by aromatherapy massage and auditory stimulation resulting from application of NMT can cause deep brain stimulation and lead to activation of the reticular activating system and more complicated pathways related to cognition and emotion. Also, aromatherapy massage and NMT may give additional activation in the bilateral superior temporal gyri and other anatomical sites, where a neural network with areas being involved in cognition and emotion exists leading to improvement of cognitive functions. ${ }^{[57]}$

Findings of the current study are supported by Alam et al. $(2016),{ }^{[48]}$ they stated that the mean scores of the RLA scale increased more significantly in the study group after application of sensory stimulation program than the control group. In addition, the results of the present study are in concordance with Castro et al. (2015), ${ }^{[17]}$ who studied the effect of using music therapy as an auditory stimulation on the cognitive functions recovery. They concluded that music has a beneficial effect on mean score of RLA scale of patients with disorders of consciousness in the study group more than in the control group.

Also, findings of Kaewsriwong et al. (2015) $)^{[31]}$ were in line with the current study findings. They explored the effect of sensory stimulation on cognitive functions recovery among the TBI patients. They found that the mean scores of RLA 
were significantly increased among TBI cases after implementation of sensory stimulation. Additionally, Okumura et al. (2014) $)^{[58]}$ investigated the changes in brain activity which reflect cognitive function recovery of TBI patients using functional Magnetic Resonance Imaging following music stimulation. They reported higher activation in the bilateral superior temporal gyri in the intervention group as compared to the control group, suggesting an impact of the music stimulation on the TBI patients' cognitive function recovery.

Another study conducted by Wu et al. (2014) ${ }^{[24]}$ reported that the plasma brain-derived neurotrophic factor (BDNF) levels were significantly higher in aromatherapy massage group than in the control group. Elevating the BDNF levels are associated with better brain plasticity and cognitive function. On the other hand, findings of the current study are contradicted by Moattari and colleagues (2016), ${ }^{[42]}$ who reported that there were no significant differences in the mean scores of RLA scale among TBI patients in the study group compared to the control after application of sensory stimulation.

The findings of the present study showed that the mean scores of basic cognitive sensory recovery evaluated by the WNSSP scale were increased significantly in patients of the study group after integrative nursing practices application compared to the control group. This favorable change may be justified by environmental enrichment. Providing an enriched environment via application of NMT, massage and aromatherapy are associated with prevention of sensory deprivation and enhancing the brain plasticity and cognitive sensory recovery. As the greater and richer the stimulation, the better are the prospects of sensory recovery. ${ }^{[12]}$

The results of the present study are supported by Chuaykarn and Jitpanya (2017) $)^{[57]}$ and Moattari et al. (2016) ${ }^{[42]}$ who noticed that the mean of WNSSP scores for TBI patients who exposed to sensory stimulation were significantly elevated than those who did not exposed. Moreover, the findings of present study are in line with Mandeep et al. (2013), ${ }^{[59]}$ who studied the efficacy of early intervention of coma arousal therapy on TBI patients with low GCS. They recorded that coma arousal therapy had significant effect on basic cognitive sensory recovery in the intervention group when compared to the control group.

Moreover, the present study findings are in congruent with the findings of Kaewsriwong et al. (2015) ${ }^{[31]}$ who reported the total mean scores of the sensory modality assessment and rehabilitation technique (SMART) scale were significantly increased among TBI cases after implementation of sensory stimulation practices. As well, Park and Davis (2016) ${ }^{[60]}$ who found that TBI patients who received auditory stimulation had a significant improvement in mean sensory stimulation assessment measure (SSAM) score over their baseline.

Also, the findings of the present study revealed that the mean scores of arousal, auditory, communication, visual, tactile and olfactory responses among patients in the study group were improved significantly from the first implementation of integrative nursing practices. This improvement among the study group can be explained by the effect of multimodal sensory stimulation resulting from application of NMT, massage and lavender oil. This explanation is in agreement with a concept given by Keller et al. (2007), ${ }^{[61]}$ they stated that application of multimodal stimulation increases the autonomic nervous system activity, confirming the fact that they have an effect on the reticular activating system and brain plasticity.

Findings of Alam et al. (2016) ${ }^{[48]}$ are in agreement with the findings of the current study. They reported that all mean scores of arousal, auditory, communication, visual, tactile and olfactory responses of the study group were significantly increased after application of stimulation program for TBI patients compared with the baseline scores.

Also, findings of the current study illustrated that the mean scores of LOC, LCF, and basic cognitive sensory recovery remained almost unchanged and did not show any significant improvement among patients in the control group. These findings may be contributed to lack of adequate and repetitive stimulation that TBI patients had been received in the study setting. Combination of little and eventual monotony stimulation from the simple conventional care was given to the TBI patients in the control group with sensory deprivation that results from decrease LOC following severe TBI usually associated with slow cognitive functions recovery in severe TBI patients.

These findings are congruent with the findings of Urbenjaphol et al. (2009) ${ }^{[62]}$ who found that TBI patients who did not go through sensory stimulation program in the control group did not show any significant improvement in the mean scores of GCS or SMART scales. In contrast with Elhady and colleagues ${ }^{[35]}$ study who illustrated that the mean scores of GCS and RLA scales were increased significantly in the control group after applying conventional nursing care by CCNs.

Based on the results of the present study, it can be noted that applying integrative nursing practices is considered a nursing priority for TBI patients to enhance cognitive recovery after severe TBI. Dependence on traditional and supportive nursing care to severe TBI patients is associated with poor outcomes. Therefore, CCNs should play a more meaning- 
ful and active role in implementing the integrative nursing practices in the severe TBI patients' plan of care.

\section{Conclusion}

Based on the current study findings, it can be concluded that implementation of integrative nursing practices (NMT, massage, and aromatherapy using lavender oil) has been enhanced cognitive recovery among severe TBI patients. It is associated with significant improvement of consciousness and arousal level, patients' behaviors that are present in each level of cognitive functions recovery and all basic cognitive sensory recovery scores (attention, auditory, expressive communication, visual, tactile, and olfactory response).

\subsection{Recommendations}

Integrative nursing practices should be a part of CCNs' daily practices while caring for patients with severe TBI. These practices should not be started before 72 hours post TBI to ensure hemodynamic stability of severe TBI patients. Nurses should assess LOC, LCF, and basic cognitive sensory recovery routinely in ICU before and after each application of integrative nursing practices to monitor patients' cognitive status. In addition to focusing on the vital role of the CCNs in enhancing the TBI patients' cognitive recovery using integrative nursing practices in the undergraduate critical care nursing courses. Besides, in-service educational programs and workshops should be conducted to raise CCNs' awareness regarding the safe use of different types of the integrative nursing practices for TBI patients with cognitive impairment. Replication of this study on large sample is needed to allow generalization of the findings and confirm the effect of the intervention. Future researches should be conducted to evaluate of the effect of integrative nursing practices on cognitive recovery among vegetative TBI patients.

\subsection{Limitations}

This study was conducted in four ICUs only from one geographical area and therefore the results cannot be generalized and the sample size does not represent all population. The limited number of severe TBI patients with the pre-determined inclusion and exclusion criteria doubled the pre-estimated period of data collection.

\section{CONFLICTS OF INTEREST Disclosure}

The authors declare that there is no conflict of interest.

\section{REFERENCES}

[1] Hickey JV. The Clinical Practice of Neurological and Neurosurgical Nursing. 7th ed. Philadelphia, PA: Wolters Kluwer/Lippincott Williams \& Wilkins; 2014.

[2] Stephens JA, Williamson KN, Berryhill ME. Cognitive Rehabilitation After Traumatic Brain Injury: A Reference for Occupational Therapists. Ooccupation, participation and health. 2015; 35(1): 5-22. PMid:26623474 https://doi.org/10.1177/15394492145617 65

[3] Walle TA, Tiruneh BT, Bashah DT. Prevalence of head injury and associated factors among trauma patients visiting surgical emergency department of Gondar University Referral Hospital, Northwest Ethiopia 2016. Across-sectional study. International Journal of Africa Nursing Sciences. 2018; 9(2): 57-61. https://doi .org/10.101 6/j.ijans.2018.08.002

[4] Peebles P, Cruz S. A primer on traumatic brain injury for nursing faculty. Journal of Professional Nursing. 2018; 34(1): 14-9. PMid:30527698 https://doi.org/10.1016/j . profnurs. 201 8.01 .002

[5] GBD. Global, regional, and national burden of traumatic brain injury and spinal cord injury, 1990-2016: a systematic analysis for the Global Burden of Disease Study 2016. The Lancet Neurology. 2019; 18(1): 56-87. https://doi.org/10.1016/S1474-4 $422(18) 30415-0$

[6] World Health Organization (WHO). World Health Statistics. Luxembourg: WHO Library Cataloguing-in-Publication Data; 2015.

[7] Oyesanya TO, Thomas MA, Brown RL, et al. Nurses' Beliefs About Caring for Patients with Traumatic Brain Injury. Western
Journal of Nursing Research. 2016; 38(9): 1114-38. PMid:26955844 https://doi.org/10.1177/0193945916636629

[8] Rabinowitz AR, Levin HS. Cognitive sequelae of traumatic brain injury. The Psychiatric clinics of North America. 2014; 37(1): 111. PMid:24529420 https://doi.org/10.1016/j.psc. 2013.1 1.004

[9] Stenberg M, Godbolt AK, Nygren De Boussard C, et al. Cognitive Impairment after Severe Traumatic Brain Injury, Clinical Course and Impact on Outcome: A Swedish-Icelandic Study. Behavioural Neurology. 2015; 2015: 680308. PMid:26783381 https: //doi.org/10.1155/2015/680308

[10] Tran LV. Understanding the pathophysiology of traumatic brain injury and the mechanisms of action of neuroprotective interventions. Journal of Trauma Nursing. 2014; 21(1): 30-5. PMid:24399316 https://doi.org/10.1097/JTN.0000000000000026

[11] Drake DF, Hudak AM, Robbins W. Integrative Medicine in Traumatic Brain Injury. Physical medicine and rehabilitation clinics of North America. 2017; 28(2): 363-78. PMid:28390519 https: //doi.org/10.1016/j.pmr.2016.12.011

[12] Sharma N, Classen J, Cohen LG. Neural plasticity and its contribution to functional recovery. Handbook of Clinical Neurology. 2013; 110: 3-12. PMid:23312626 https://doi .org/10.1016/B978-0 -444-52901-5.00001-0

[13] Becker C. Nursing care of the brain injury patient on a locked neurobehavioral unit. Rehabilitation nursing: the Official Journal of the Association of Rehabilitation Nurses. 2012; 37(4): 171-5. PMid:22744988 https://doi.org/10.1002/rnj.50

[14] Gunnarsdottir PJ, Koithan M, Kristofersson GK. Integrative Nursing Principles in Action: A Summary From the First International Inte- 
grative Nursing Symposium. Creative Nursing. 2015; 21(4): 222-5. PMid:26731921 https://doi .org/10.1891/1078-4535.21.4 .222

[15] Papathanassoglou E, Park T. To put the patient in the best condition: integrating integrative therapies in critical care. Nursing in Critical Care. 2016; 21(3): 123-6. PMid:27041347 https://doi .org/10 $.1111 / \mathrm{nicc} .12243$

[16] Gnatta JR, Kurebayashi LF, Turrini RN, et al. Aromatherapy and nursing: historical and theoretical conception. Revista da Escola de Enfermagem da U S P. 2016; 50(1): 130-6. PMid:27007430 https://doi.org/10.1590/S0080-623420160000100017

[17] Castro M, Tillmann B, Luaute J, et al. Boosting Cognition with Music in Patients with Disorders of Consciousness. Neurorehabilitation and Neural Repair. 2015; 29(8): 734-42. PMid:25650390 https://doi.org/10.1177/1545968314565464

[18] Davis AE, Gimenez A. Cognitive-behavioral recovery in comatose patients following auditory sensory stimulation. Journal of Neuroscience Nursing. 2003; 35(4): 202-9, 14. PMid:12942654 https: //doi.org/10.1097/01376517-200308000-00006

[19] Hegde S. Music-based cognitive remediation therapy for patients with traumatic brain injury. Frontiers in Neurology. 2014;5: 34 PMid:24715887 https://doi.org/10.3389/fneur.2014.000 34

[20] YekeFallah L. Tactile stimulation improves consciousness and vital signs in patients with traumatic brain injury:clinical trial. Nursing Critical Care. 2018; 13(6): 18-22. https://doi.org/10.1097/ 01. CCN. 0000546310.00168 .30

[21] Hatefi M, Jaafarpour M, Khani A, et al. The Effect of Whole Body Massage on the Process and Physiological Outcome of Trauma ICU Patients: A Double-Blind Randomized Clinical Trial. Journal of Clinical and Diagnostic Research. 2015; 9(6): UC05-8.

[22] Azami H, Khaledi B, Rezaei M, et al. The impacts of short-term foot massage on mean arterial pressure of neurosurgical patients hospitalized in intensive care units. Iran Journal of Critical Care Nursing. 2015; 8(3): 133-42.

[23] Kohanpour MA, Peeri M, Azarbayjani MA. The effects of aerobic exercise with lavender essence use on cognitive state and serum brain-derived neurotrophic factor levels in elderly with mild cognitive impairment. Journal of Herbmed Pharmacology. 2017; 6(2): $80-4$

[24] Wu JJ, Cui Y, Yang YS, et al. Modulatory effects of aromatherapy massage intervention on electroencephalogram, psychological assessments, salivary cortisol and plasma brain-derived neurotrophic factor. Complementary Therapies in Medicine. 2014; 22(3): 456-62. PMid:24906585 https://doi.org/10.1016/j.ctim.2014.04 .001

[25] Sayorwan W, Siripornpanich V, Piriyapunyaporn T, et al. The effects of lavender oil inhalation on emotional states, autonomic nervous system, and brain electrical activity. Journal of the Medical Association of Thailand. 2012; 95(4): 598-606.

[26] Allard ME, Katseres J. Using Essential Oils to Enhance Nursing Practice and for Self-Care. The American Journal of Nursing. 2016 116(2): 42-9. PMid:26771667 https://doi.org/10.1097/01.N AJ .0000480495.18104.db

[27] Kreitzer MJ. Integrative nursing: application of principles across clinical settings. Rambam Maimonides Medical Journal. 2015; 6(2): e0016. PMid:25973268 https://doi.org/10.5041/RMMJ.102 00

[28] Albaqawi HM, Butcon VR, Molina RR. Awareness of holistic care practices by intensive care nurses in north-western Saudi Arabia. Saudi Medical Journal. 2017; 38(8): 826-31. PMid:28762435 https://doi.org/10.15537/smj.2017.8.20056
[29] Kramlich D. Strategies for Acute and Critical Care Nurses Implementing Complementary Therapies Requested by Patients and Their Families. Critical Care Nurse. 2016; 36(6): 52-8. PMid:27908946 https://doi.org/10.4037/ccn2016974

[30] Cusick A, Lannin NA, Hanssen R, et al. Validating the Western Neuro Sensory Stimulation Profile for patients with severe traumatic brain injury who are slow-to-recover. Australian Occupational Therapy Journal. 2014; 61(4): 276-83. PMid:24809954 https://doi.org/10.1111/1440-1630.12128

[31] Kaewsriwong S, Sukonthasarn A, Wangsrikhun S, et al. Sensory Stimulation Process and Cognitive Function among Persons with Traumatic Brain Injury: A Case Study. Pacific Rim International Journal of Nursing Research. 2015; 19(1): 45-57.

[32] Uyar M, Akin Korhan E. The effect of music therapy on pain and anxiety in intensive care patients. Agri. 2011; 23(4): 139-46. PMid:22290677 https://doi.org/10.5505/agri.2011.9469 5

[33] Ozlu ZK, Bilican P. Effects of Aromatherapy Massage on the Sleep Quality and Physiological Parameters of Patients in a Surgical Intensive Care Unit. African Journal of Traditional, Complementary, and Alternative Medicines. 2017; 14(3): 83-8. PMid:28480419 https://doi.org/10.21010/ajtcam.v14i3.9

[34] Cho E, Lee M, Hur M. The Effects of Aromatherapy on Intensive Care Unit Patients' Stress and Sleep Quality: A Nonrandomised Controlled Trial. Evidence-based Complementary and Alternative Medicine. 2017; 2017: 2856592. PMid:29375641 https : //doi.org/10.1155/2017/2856592

[35] Elhady M, EL-Sousi A, Othman S. The effect of implementing a structured simulation program on altered consciousness of critically ill patients. Alexandria Scientific Nursing Journal. 2011; 12(1): 3556.

[36] Jamaati H, Azimi A, Ebadi A, et al. Therapeutic Effect of Massage on the Patients in Intensive Care Unit. Archives of Critical Care Medicine. 2015; 1(1): 1-8. https ://doi.org/10.5812/accm.5 19

[37] Wijdicks EF, Bamlet WR, Maramattom BV, et al. Validation of a new coma scale: The FOUR score. Annals of Neurology. 2005; 58(4): 585-93. PMid:16178024 https ://doi.org/10.1002/ana. 2061 1

[38] Hagen C, Malkmus D, Durham P. Ranchos Los Amigos Level of Cognitive Functioning Scale. Downet, CA: Communications Disorders Services, Ranchos Los Amigos Hospital; 1972.

[39] Ansell BJ, Keenan JE. The Western Neuro Sensory Stimulation Profile: a tool for assessing slow-to-recover head-injured patients. Archives of Physical Medicine and Rehabilitation. 1989; 70(2): 104 8.

[40] Iyer VN, Mandrekar JN, Danielson RD, et al. Validity of the FOUR score coma scale in the medical intensive care unit. Mayo Clinic Proceedings. 2009; 84(8): 694-701. https://doi.org/10.4065/ 84.8 .694

[41] McCauley SR, Wilde EA, Kelly TM, et al. The Neurological Outcome Scale for Traumatic Brain Injury (NOS-TBI): II. Reliability and convergent validity. Journal of Neurotrauma. 2010; 27(6): 991-7. PMid:20210595 https://doi.org/10.1089/neu.2009.1195

[42] Moattari M, Alizadeh Shirazi F, Sharifi N, et al. Effects of a Sensory Stimulation by Nurses and Families on Level of Cognitive Function, and Basic Cognitive Sensory Recovery of Comatose Patients With Severe Traumatic Brain Injury: A Randomized Control Trial. Trauma Monthly. 2016; 21(4): e23531. PMid:28180120 https://doi.org/10.5812/traumamon. 23531

[43] Jagan S, Park T, Papathanassoglou E. Effects of massage on outcomes of adult intensive care unit patients: a systematic review. 
Nursing in Critical Care. 2019; 24(6): 414-29. PMid:30829459 https://doi.org/10.1111/nicc.12417

[44] Vahedian-Azimi A, Ebadi A, Asghari Jafarabadi M, et al. Effect of Massage Therapy on Vital Signs and GCS Scores of ICU Patients: A Randomized Controlled Clinical Trial. Trauma Monthly. 2014; 19(3): e17031. PMid:25337518 https ://doi.org/10.5812/traumamo n. 17031

[45] Varghese R, Chakrabarty J, Menon G. Nursing Management of Adults with Severe Traumatic Brain Injury: A Narrative Review. Indian Journal of Critical Care Medicine. 2017; 21(10): 684-97. PMid:29142381 https://doi.org/10.4103/ijccm.IJCCM_23 3_17

[46] Oyesanya TO, Brown RL, Turkstra LS. Caring for Patients with traumatic brain injury: a survey of nurses' perceptions. Journal of Clinical Nursing. 2017; 26(11-12): 1562-74. PMid:27346166 https://doi.org/10.1111/jocn. 13457

[47] Rebecca L, Iris R. Integrative Nursing Management of Cognitive Impairment In: BellKreitzer MJ, Koithan M., editors. Integrative Nursing. 2nd ed. New York, NY: Oxford University Press; 2014. p. 300-13.

[48] Alam Z, Elsaay O, Weheida S, et al. Effect of Sensory and Motor Stimulation Program on Clinical Outcomes of Patients with Moderate Head Injury. IOSR Journal of Nursing and Health Science. 2016; 5(5): 24-36.

[49] El-Fiki A, El Falaky O. Evaluation of neurotrauma in motorcyclerelated accidents at a tertiary hospital in Egypt. The Egyptian Journal of Neurology, Psychiatry and Neurosurgery. 2016; 53: 244. https://doi.org/10.4103/1110-1083.202385

[50] Altenmüller E, Schlaug G. Neurobiological aspects of neurologic music therapy. Music ands Medicine. 2013; 5(2): 210-6. PMid:25774137 https://doi.org/10.3389/fpsyg.2014.01185

[51] Padilla R, Domina A. Effectiveness of sensory stimulation to improve arousal and alertness of people in a coma or persistent vegetative state after traumatic brain injury: A systematic review. American Journal of Occupational Therapy. 2016; 70(3): 1-8. PMid:27089287 https://doi.org/10.5014/ajot.2016.021022

[52] Megha M, Harpreet S, Nayeem Z. Effect of frequency of multimodal coma stimulation on the consciousness levels of traumatic brain injury comatose patients. Brain Injury. 2013; 27(5): 570-7. PMid:23473238 https://doi.org/10.3109/02699052.2013.767937
[53] Sun J, Chen W. Music therapy for coma patients: preliminary results. European Review for Medical and Pharmacological Sciences. 2015; 19(7): 1209-18.

[54] Cevik K, Namik E. Effect of Auditory Stimulation on the Level of Consciousness in Comatose Patients Admitted to the Intensive Care Unit: A Randomized Controlled Trial. Journal of Neuroscience Nursing. 2018; 50(6): 375-80. PMid:30407969 https: //doi.org/10.1097/JNN.0000000000000407

[55] Jalil A, Mohammad A, Mohammad M, et al. The effect of foot massage on the consciousness levels in comatose patients with brain injury hospitalized in intensive care unit (icu): a randomised control trial. Knowledge and Health. 2015; 10(3): 25-30.

[56] Cheng L, Cortese D, Monti MM, et al. Do Sensory Stimulation Programs Have an Impact on Consciousness Recovery? Frontiers in Neurology. 2018; 9: 826. PMid:30333789 https://doi.org/10 $.3389 /$ fneur. 2018.00826

[57] Chuaykarn U, Jitpanya C. Effects of Two Sensory Stimulation Models on Recovery in Adults with Severe Traumatic Brain Injury. International Journal of Medical Research \& Health Sciences. 2017; 6(8): 69-74.

[58] Okumura Y, Asano Y, Takenaka S, et al. Brain activation by music in patients in a vegetative or minimally conscious state following diffuse brain injury. Brain Injury. 2014; 28(7): 944-50. PMid:24655034 https://doi.org/10.3109/02699052.2014.888477

[59] Mandeep, Chitkara N, Goel S, et al. Traumatic head injury: Early intervention by coma arousal therapy. The Indian Journal of Neurotrauma. 2013; 10(1): 13-8. https://doi.org/10.1016/j.ijnt .2013 .05 .004

[60] Park S, Davis AE. Effectiveness of direct and non-direct auditory stimulation on coma arousal after traumatic brain injury. International Journal of Nursing Practice. 2016; 22(4): 391-6. PMid:27241789 https://doi.org/10.1111/ijn.12448

[61] Keller I, Hulsdunk A, Muller F. The influence of acoustic and tactile stimulation on vegetative parameters and EEG in persistent vegetative state. Functional Neurology. 2007; 22(3): 159-63.

[62] Urbenjaphol P, Jitpanya C, Khaoropthum S. Effects of the Sensory Stimulation Program on Recovery in Unconscious Patients With Traumatic Brain Injury. Journal of Neuroscience Nursing. 2009; 41(4): E10-E6. http://doi.org/10.1097/JNN . Ob013e3181a23e94 